\title{
Numerical Solution of Tumor-immune Model including small Molecule Drug by Multi-step Differential Transform Method
}

\author{
Biplab Dhar ${ }^{1}$, Praveen Kumar Gupta ${ }^{2}$ \\ ${ }^{1,2}$ Department of Mathematics, National Institute of Technology Silchar, Assam-788010, India \\ 1'bipcoparya@gmail.com, ${ }^{2}$ pkguptaitbhu@gmail.com
}

\begin{abstract}
In this article, we have described a mathematical model for tumor-immune system with impact of small molecules drug, and solved by multi step differential transform method. The mathematical model has four compartments; population of tumor cell, CD8 T-killer cell, CD4 T-helper cell and amount of drug in the blood stream. Calculations are presented to verify the theoretical results so obtained for tumor free equilibrium point. The model is fit for removing tumor with passage of time irrespective of the initial population or size of the tumor.
\end{abstract}

Key words: Mathematical model, Tumor-immune system, Molecule drug, MsDTM.

\section{INTRODUCTION}

Tumor is an endemic around the globe, and yet not much is known in about its development and demolition. The tumor cells spread to body by the phenomenon called metastasis, and they can be classified into two kinds: non-cancerous and cancerous. However, dangerous tumor is a multi factorial sickness, the hazard elements of which incorporate utilization of tobacco items, unfortunate eating routine, air contamination and so on.

CD4 T-helper cells and CD8 T-killer cells are two most insusceptible cells for securing against malignant cells. CD4 T-helper cells generally help all types of body cells of the immune system in contamination, and CD8 T-killer cells protect and set out infected cells. According to law of mass action, as stated by Sharma [15], CD4 T-helper cells are transformed into CD8 T-killer cells; it may be direct contact with them, or other case interaction with cytokines generated by the CD4 T-helper cells. At some point, the immune system neglects to battle such tumor cells because of conceivable after reasons, (i) tumor-tolerance; and (ii) regardless of whether the immune systems perceive tumor cells the safe reaction may not to be sufficiently strong to eradicate them. Thus, the immune system needs external effective measure to eradicate the tumor cells.
There are different tumor treatment such as; surgery, radiotherapy, immunotherapy, targeted treatment, hormone treatment, chemotherapy (CTx) and many more. Targeted chemotherapy is a medication that piece the dynamic idea of tumor by meddling with particular particles which are associated with the development and spread of the growth, though standard CTx follow up on all rapidly separating solid and cancerous cells. In addition to that, experimental results indicate that tumor cells are killed $91 \%$ more by targeted CTx than CTx [12]. A particular type of targeted CTx called "small-molecule drugs" can reach tumor cells very easily as it has low molecular weight. The moment this drug enters the tumor cells, it attack the other molecules which cause malignancy. In the year 2006, Ganguly [5] mentioned that with efficacies of drugs towards cell populations a chemotherapeutic drug can be designed.

In 2013, Sharma [14] introduced a tumor-growth model considering cytotoxic T-cells and controlling the number of tumor cells and drug administered. In 2017, Liu [12] modified a tumor-immune model with the help of Pillis et. al [3] by taking monoclonal antibodies into account. These drugs have large molecular weight in comparison to small molecule drug and therefore unable to get into the tumor cells eventually forming a layer outside the tumor region. But Liu failed to eradicate the large tumor cells population $\left(10^{7}\right)$ from the system. In the same year, Sharma [15] presented another tumor growth model with the help of tumor-immune communications and immunotherapeutic / chemotherapeutic drugs, to reduce the number of tumor cells and supervision of drugs. There are so many works in the literature on tumor growth models, which can be found in $[1,2,6,8,9,11,12$, and 16].

The aim of this work is to find an adequate condition and to carry out the dynamics of the model in order to eradicate the tumor cells of any size of population.

\section{THE MODEL}

\subsection{Model Description}

Here, we build a mathematical model for tumor growth including with immune response as well as small molecule drug, which is described by the following set of ODEs: 
Biplab Dhar et al., International Journal of Advanced Trends in Computer Science and Engineering, 8(5),September - October 2019, 1802 - 1807

$$
\begin{aligned}
& \dot{T}=r_{1} T\left(1-b_{1} T\right)-\alpha T E_{k}-f T C, \\
& \dot{E}_{k}=\beta E_{k} E_{h}-\alpha_{k} T E_{k}-d E_{k}-f_{1}(1-\mu) E_{k} C, \\
& \dot{E}_{h}=r_{2} E_{h}\left(1-b_{2} E_{h}\right)-\beta E_{k} E_{h}-f_{2}(1-\mu) E_{h} C, \\
& \dot{C}=v_{0}-\gamma C,
\end{aligned}
$$

with initial conditions,

$$
T(0)=T_{0}, E_{k}(0)=E_{k 0}, E_{h}(0)=E_{h 0}, C(0)=C_{0},
$$

where $T(t), E_{k}(t), E_{h}(t)$ are the population of tumor cells, CD8 T-killer cells, CD4 T-helper cells, respectively, and $C(t)$ is the amount of small molecule drug in the blood stream at time $t$.

\begin{tabular}{|c|c|c|}
\hline $\begin{array}{c}\text { Paramete } \\
\mathbf{r}\end{array}$ & Value & Units \\
\hline$r_{1}$ & 0.46 & per day \\
\hline$r_{2}$ & 0.0246 & per day \\
\hline$b_{1}$ & $5 \times 10^{-9}$ & per cells \\
\hline$b_{2}$ & $1.1 \times 10^{-10}$ & per cells \\
\hline$\alpha$ & $1.101 \times 10^{-7}$ & per cells per day \\
\hline$\alpha_{k}$ & $3.423 \times 10^{-10}$ & per cells per day \\
\hline$\beta$ & $6.3 \times 10^{-9}$ & per cells per day \\
\hline$\gamma$ & 0.01 & per day \\
\hline$f$ & 0.9 & per day \\
\hline$f_{1}$ & $2 \times 10^{-10}$ & per day \\
\hline$f_{2}$ & $10^{-4}$ & per day \\
\hline$d$ & 0.0413 & per day \\
\hline$v_{0}$ & {$[0,0.5]$} & per day \\
\hline$\mu$ & {$[0,1]$} & \\
\hline
\end{tabular}

Table 1: The estimated parameter values for the proposed system (1)-(4) are taken from $[8,12,15]$.

The proposed model goes through some assumptions which are mentioned as:

i) All the parameters used in the model are positive constants and all cells are taken in small volume of tissue.

ii) The population of tumor cells and that of CD4 T-helper cells are assumed to emerge logistically with rates $r_{1}$ and $r_{2}$, respectively in the absence of CD8 T-killer cells and chemotherapeutic drug. The growth rate $r_{1}$ is also due to hazard elements stated in the section 1.

iii) The tumor cells and CD8 T-killer cells are being killed at rates $\alpha$ and $\alpha_{k}$, respectively, proportional to the product densities of tumor cells and CD8 T-killer cells. Also $d$ is per capita degradation rate of CD8 T-killer cells.

iv) Due to law of mass action CD4 T-helper cells transform to CD8 T-killer cells with rate $\beta$.

v) After the administration of drug into the blood stream it destroys tumor cells, CD8 T-killer cells and CD4 T-helper cells with rates $f, f_{1}(1-\mu)$ and $f_{2}(1-\mu)$, respectively. Here, $\mu$ is the efficacy term. In pharmacology, efficacy is the maximum response achievable from an applied or dosed drug. vi) The drug dosage is denoted by $v_{0}$ and $\gamma$ is the per capita decay rate of drug.

\subsection{Basic properties}

Theorem 1. (see [4]) The solution of the system (1)-(4) with initial conditions (5) exists in the interval $[0, \xi]$, where $0 \leq \xi<$ $\infty$, i.e., $T(t) \geq 0, E_{k}(t)>0, E_{h}(t)>0, C(t)>0$ for all $t \geq 0$.

Theorem 2. (see [4]) The feasible region $S$ is defined by

$$
D=\left\{\left(T, E_{k}, E_{h}, C\right) \in \mathfrak{R}_{+}^{4} \mid T \geq 0, E_{k}>0, E_{h}>0, C \geq 0\right\}
$$

is a positive invariant set of the system (1)-(4) and is also bounded.

\section{MODEL ANALYSIS}

From Theorem 1 and Theorem 2, $D$ is a positive invariant set of the proposed system. Hence, we refer the entire region $D$ as a "Global" domain. The system (1)-(4) has four equilibrium points as follows:

i) trivial equilibrium: $S_{1}\left(0,0,0, \frac{v_{0}}{\gamma}\right)$

ii) dead equilibrium: $S_{2}\left(\bar{T}, 0,0, \frac{v_{0}}{\gamma}\right), S_{3}\left(\bar{T}, 0, \bar{E}_{h}, \frac{v_{0}}{\gamma}\right)$,

iii) tumor-free equilibrium: $S_{4}\left(0,0, \bar{E}_{h}, \frac{v_{0}}{\gamma}\right)$, $S_{5}\left(0, E_{k_{5}}, E_{h_{5}}, \frac{v_{0}}{\gamma}\right)$,

iv) co-existing equilibrium: $S^{*}\left(T^{*}, E_{k}^{*}, E_{h}^{*}, \frac{v_{0}}{\gamma}\right)$,

where, $\bar{T}=\frac{r_{1} \gamma-f v_{0}}{b_{1} r_{1} \gamma}>0, \quad \bar{E}_{h}=\frac{r_{2} \gamma-f_{2}(1-\mu) v_{0}}{b_{2} r_{2} \gamma}>0$,

$E_{h_{5}}=\frac{f_{1}(1-\mu) v_{0}+d \gamma}{\beta \gamma}>0, \quad E_{k_{5}}=\frac{r_{2} \beta \gamma-d b_{2} r_{2} \gamma-f_{2}(1-\mu) \beta v_{0}-b_{2} f_{1}(1-\mu) r_{2} v_{0}}{\beta^{2} \gamma}>0$,

$T^{*}=\frac{b_{2} r_{2} \alpha\left(f_{1}(1-\mu) v_{0}+d \gamma\right)-\beta\left(r_{2} \alpha \gamma+f \beta v_{0}-f_{2}(1-\mu) \alpha v_{0}-r_{1} \beta \gamma\right)}{\gamma\left(b_{1} r_{1} \beta^{2}-b_{2} r_{2} \alpha \alpha_{k}\right)}>0$,

$E_{k}^{*}=\frac{b_{2} r_{2} \alpha_{k}\left(f v_{0}-r_{1} \gamma\right)-b_{1} r_{1}\left(b_{2} r_{2} f_{1}(1-\mu) v_{0}+f_{2}(1-\mu) \beta v_{0}+d b_{2} r_{2} \gamma-r_{2} \beta \gamma\right)}{\gamma\left(b_{1} r_{1} \beta^{2}-b_{2} r_{2} \alpha \alpha_{k}\right)}>0$, and

$E_{h}^{*}=\frac{f_{2}(1-\mu) \alpha \alpha_{k} v_{0}+b_{1} r_{1} \beta\left(f_{1}(1-\mu) v_{0}+d \gamma\right)-\alpha_{k}\left(f \beta v_{0}+r_{2} \alpha \gamma-r_{1} \beta \gamma\right)}{\gamma\left(b_{1} r_{1} \beta^{2}-b_{2} r_{2} \alpha \alpha_{k}\right)}>0$.

\subsection{Biological clarification of equilibrium points}

For $S_{1}$, each of the cells gets pulverized and it implies that the treatment is not worthwhile. In $S_{2}$ and $S_{3}$, tumor cells exist which implies that after the drug injection either of the T cells or CD8 T-killer and CD4 T-helper cells are obliterated, however the tumor cells holds on. Henceforth, the stability of this point isn't helful for treatment. In $\mathrm{S}_{4}$ and $\mathrm{S}_{5}$, tumor cells are eradicated after the drug injection. We also see that either CD8 T-killer cells or CD4 T-helper cells gets destroyed but not both at a time. Hence, stability of this point is useful for the therapy. Lastly in $S^{*}$, all the cells exist but if this equilibrium point is stable then tumor cells will remain as a constant in the system and will neither grow nor shrink.

3.2 Local stability analysis of $S_{4}$ and $S_{5}$

Theorem 3. (see [4]) The system (1)-(4) consists the tumor free equilibrium point $S_{4}$ and it also locally asymptotically stable if $v_{0} f_{1}(1-\mu)>\gamma\left(\beta \bar{E}_{h}-d\right)$, and one more tumor free equilibrium $S_{5}$ for the system (1)-(4) exists and it is locally asymptotically stable if $v_{0} f>\gamma\left(r_{1}-\alpha E_{k_{5}}\right)$. 
Biplab Dhar et al., International Journal of Advanced Trends in Computer Science and Engineering, 8(5),September - October 2019, 1802 - 1807

\section{DTM AND Ms-DTM}

\subsection{Solution by DTM}

The detailed explanation of DTM can be found in works done by Gupta [7] and Kumari et al. [10]. Here, we will try to give a brief definition of DTM.

Let us assume a general equation of $n^{\text {th }}$ order O.D.E

$$
f\left(t, x, x^{\prime}, x^{\prime \prime}, \ldots, x^{(n)}\right)=0
$$

with initial conditions $x^{(l)}=d_{l}$ and $l=0,1, \ldots,(n-1)$. Let $x(t)$ be regular in the domain $\Gamma=[0, \bar{T}]$ and let $t=t_{0}$ be any point in $\Gamma$. Then this function $x(t)$ can be represented by a power series centering at $t_{0}$. The differential transformation of the $l t h$ derivative of a function $x(t)$ is defined as follows:

$$
X(l)=\frac{1}{l !}\left[\frac{d^{i}}{d t^{i}} x(t)\right]_{t=t_{0}}, \text { for all } t \in \Gamma .
$$

In equation (7), $X(l)$ is the transformed equation of the function $x(t)$. The inverse differential transformation of $X(l)$ is presented as follows:

$$
x(t)=\sum_{l=0}^{\infty} X(l)\left(t-t_{0}\right)^{l}, \text { for all } t \in \Gamma .
$$

Thus, from equation (7) and (8), we get

$$
x(t)=\sum_{l=0}^{\infty} \frac{\left(t-t_{0}\right)^{l}}{l !}\left[\frac{d^{i}}{d t^{i}} x(t)\right]_{t=t_{0}}, \text { for all } t \in \Gamma .
$$

The function $x(t)$, in real applications, is converted to a finite series because it is assumed that $\sum_{N+1}^{\infty} X(l)\left(t-t_{0}\right)^{l}$ is negligible and so equation (8) can be written as:

$$
x(t)=\sum_{l=0}^{N} X(l)\left(t-t_{0}\right)^{l} \quad \text {, for all } t \in \Gamma .
$$

\subsection{Solution by Ms-DTM}

The Ms-DTM is an algorithm for deducing precisely approximate solutions in a sequence of intervals for system of both weak and strong non-linear differential equations. We have seen that the approximate solution of the above initial value problem (9) can be expressed as

$$
x(t)=\sum_{l=0}^{N} a_{l} t^{l}
$$

for all $t \in[0, \bar{T}]$. Now, we make the partition of full interval $[0, \bar{T}]$ into $N$ sub-intervals $\left[t_{s-1}, t_{s}\right], s=1,2, \ldots, M$ of equal length $h=\frac{\bar{T}}{M}$ with the help of the nodes $t_{s}=s . h$. The detailed Ms-DTM can be found in the work performed by Odibat [13]. We at first apply DTM to equation (9) over the interval $\left[0, t_{1}\right]$ and so obtain the following approximated solution,

$$
x_{1}(t)=\sum_{l=0}^{K} a_{1 l} t^{l}
$$

for all $t \in\left[0, t_{1}\right]$ with initial conditions $x_{1}^{(l)}(0)=b_{l}$.

For $s \geq 2$, for each subinterval $\left[t_{s-1}, t_{s}\right]$, we define the initial conditions $x_{s}^{(l)}\left(t_{s-1}\right)=x_{s}^{(l-1)}\left(t_{s-1}\right)$, afterwards we apply DTM to equation (9) over the interval $\left[t_{s-1}, t_{s}\right]$, where $t_{0}$ in equation (9) is replaced by $t_{s-1}$. This process is iterated and create a sequence of approximate analytical solutions $x_{s}(t), s=1,2$, ..., $M$ for the solution $x(t)$,

$$
x_{s}(t)=\sum_{l=0}^{K} a_{s l}\left(t-t_{s-1}\right)^{l}
$$

where, $K=\frac{N}{M}$.

In fact, the Ms-DTM assumes the following solution,

$$
x(t)=\left\{\begin{array}{cc}
x_{1}(t), & t \in\left[0, t_{1}\right] \\
x_{2}(t), & t \in\left[t_{1}, t_{2}\right] \\
\vdots & \vdots \\
x_{M}(t), & t \in\left[t_{M-1}, t_{M}\right]
\end{array}\right.
$$

\section{NUMERICAL SIMULATION}

First of all, we apply the DTM to the system (1)-(4), and get the following system of equations,

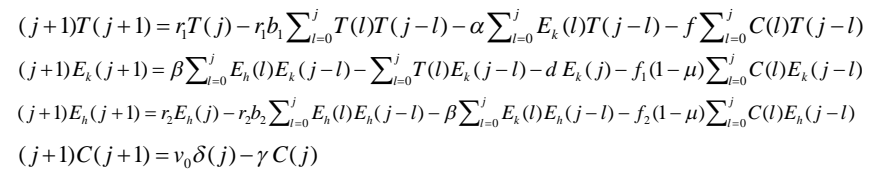

where, $T(j), E_{k}(j), E_{h}(j)$ and $C(j)$ are the differential transformation of $T(t), E_{k}(t), E_{h}(t)$ and $C(t)$, respectively. The initial conditions are given by $T(j)=T_{0}, E_{k}(j)=E_{k 0}, E_{h}(j)=E_{h 0}$ and $C(j)=C_{0}$.

According to Ms-DTM taking $N=K . M$, where we have taken $K=20$ and $M=550$, the series solution for the system (1)-(4) is given by,

$$
Y(t)=\left\{\begin{array}{cc}
\sum_{n=0}^{K} Y_{1}(n) t^{n}, & t \in\left[0, t_{1}\right] \\
\sum_{n=0}^{K} Y_{2}(n)\left(t-t_{1}\right)^{n}, & t \in\left[t_{1}, t_{2}\right] \\
\vdots & \vdots \\
\sum_{n=0}^{K} Y_{M}(n)\left(t-t_{M-1}\right)^{n}, & t \in\left[t_{M-1}, t_{M}\right]
\end{array}\right.
$$

where $T(t), E_{k}(t), E_{h}(t)$ and $C(t)$ satisfy the equation (13). Simlarly, $T_{s}(n), E_{k s}(n), E_{h s}(n)$ and $C_{s}(n)$, for $s=1,2, \ldots, M$, satisfy the iterative relations,

$$
\begin{aligned}
& T(j+1)=\frac{1}{j+1}\left[r_{1} T_{s}(j)-r_{1} b_{1} \sum_{l=0}^{j} T_{s}(l) T_{s}(j-l)-\alpha \sum_{l=0}^{j} E_{k s}(l) T_{s}(j-l)-f \sum_{l=0}^{j} C_{s}(l) T_{s}(j-l)\right] \\
& E_{k s}(j+1)=\frac{1}{j+1}\left[\beta \sum_{l=0}^{j} E_{l s s}(l) E_{k s}(j-l)-\sum_{l=0}^{j} T_{s}(l) E_{k s}(j-l)-d E_{k s}(j)-f_{1}(1-\mu) \sum_{l=0}^{j} C_{s}(l) E_{k s}(j-l)\right] \\
& E_{h s}(j+1)=\frac{1}{j+1}\left[r_{2} E_{h s}(j)-r_{2} b_{2} \sum_{l=0}^{j} E_{l s}(l) E_{h s}(j-l)-\beta \sum_{l=0}^{j} E_{k s}(l) E_{l s}(j-l)-f_{2}(1-\mu) \sum_{l=0}^{j} C_{s}(l) E_{h s}(j-l)\right] \\
& C_{s}(j+1)=\frac{1}{j+1}\left[v_{0} \delta(j)-\gamma C_{s}(j)\right]
\end{aligned}
$$

subject to

i) $T_{0}(0)=T_{0}, E_{k 0}(0)=E_{k 0}, E_{h 0}(0)=E_{h 0}, C_{0}(0)=C_{0}$, and

ii) $T_{s}(0)=T_{s-1}(0), E_{k s}(0)=E_{k s-1}(0), E_{h s}(0)=E_{h s-1}(0)$, and $C_{s}(0)=C_{s-1}(0)$.

We have considered two cases where the initial population of tumor cell are taken as $T_{0}=10^{7}$ (large tumor size) and $T_{0}=10^{5}$ (small tumor size). The initial population of CD8 T-killer cell is $E_{k 0}=3 \times 10^{5}$, CD4 T-helper cell is $E_{h 0}=3 \times 10^{5}$ [12] and the initial amount of targeted chemotherapeutic drug $C_{0}=0.50$, presented in for both the cases. This will help us to understand the behaviour of drug in the tumor prevailing system as well as in tumor recessive system.

In Figure 1 and 2, we have considered three different values for $v_{0}$, which are; 0.003 [14], 0.005 (estimated) and 0.007 (estimated). These values of $v_{0}$ do not hamper to any of the analytical findings associated with tumor free equilibrium. 
Biplab Dhar et al., International Journal of Advanced Trends in Computer Science and Engineering, 8(5),September - October 2019, 1802 - 1807

Taking the dosage as $v_{0}=0.003,0.005$ and 0.007 respectively, we encounter that number of tumor cells can be controlled and eventually the tumor free equilibrium point $\left(S_{5}\right)$ can be attained. The increase in tumor cells is observed due to low dosage of the drug into the bloodstream.

In Figure 3 and 4, we have conducted a behavioral comparison in the population of tumor cells by both Ms-DTM and DTM. We have taken $v_{0}=0.007$, and both the sizes of tumor cell and finally see that in case of Ms-DTM the population converges after sometime which is not in the case of DTM. In DTM, the population of tumor cell tends towards negative value which violates the tumor-free equilibrium point.

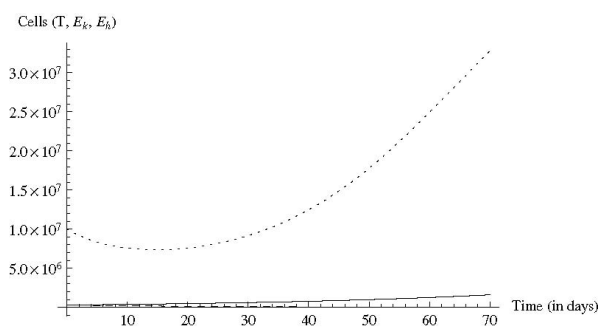

(a) $v_{0}=0.003$

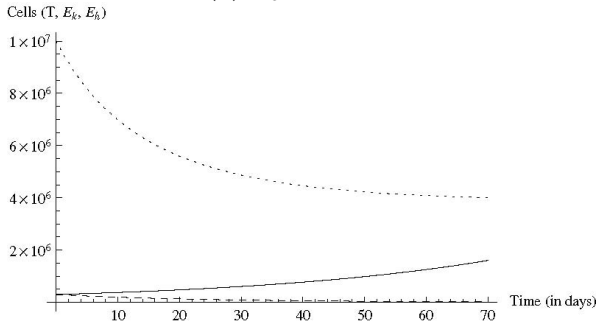

(b) $v_{0}=0.005$

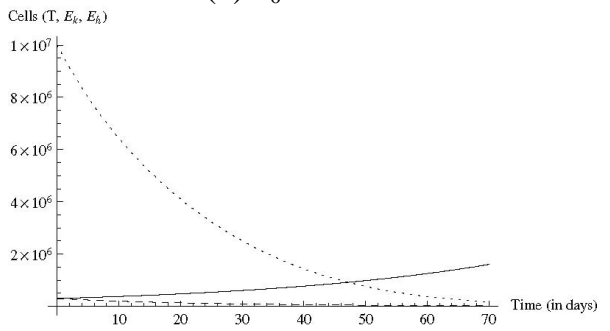

(c) $v_{0}=0.007$

Figure 1: Plot of population of large tumor cell $T(\cdots \cdots)$ CD8 T-killer cell, Ek(---), and CD4 T-helper cell, $E h(-)$ vs. time $t$.

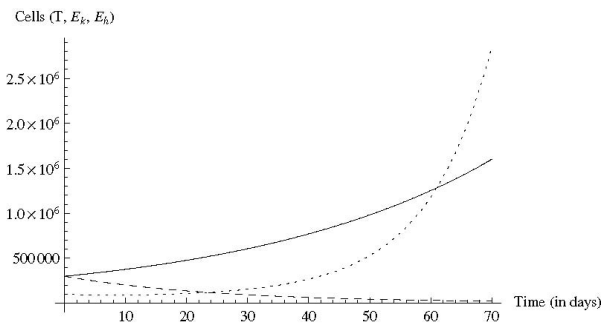

(a) $v_{0}=0.003$

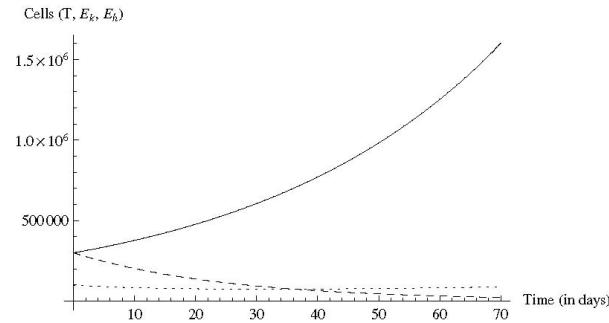

(b) $v_{0}=0.005$

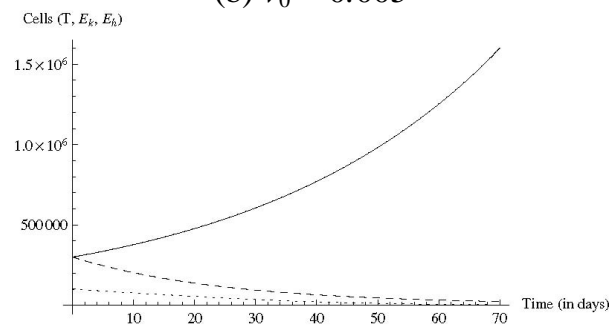

(c) $v_{0}=0.007$

Figure 2: Plot of population of small tumor cell $T(\cdot \cdots)$, CD8 T-killer cell $E k(---)$ ) and CD4 T-helper cell, $E h(-)$ vs. time $t$.

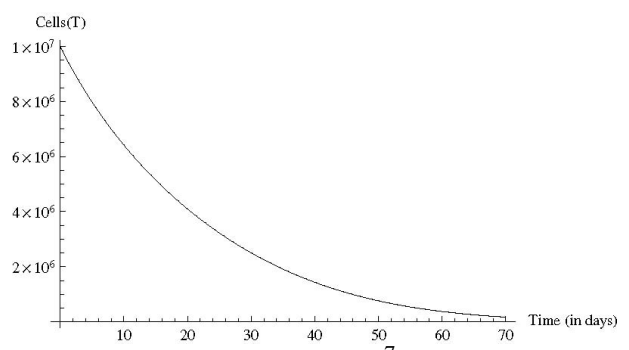

(a) $T_{0}=10^{7}$

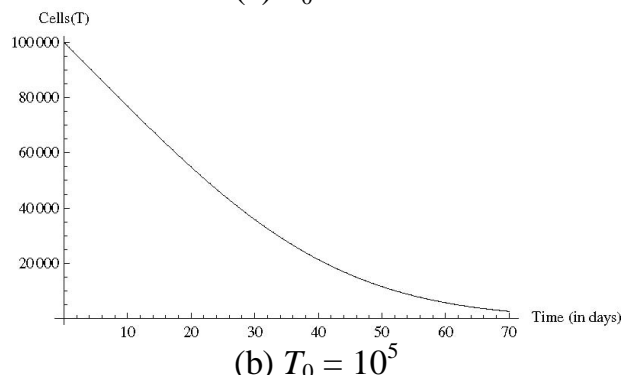

Figure 3: Plot of (a) large population, (b) small population of tumor cell vs. time $t$ for MsDTM, when $v_{0}=0.007$.

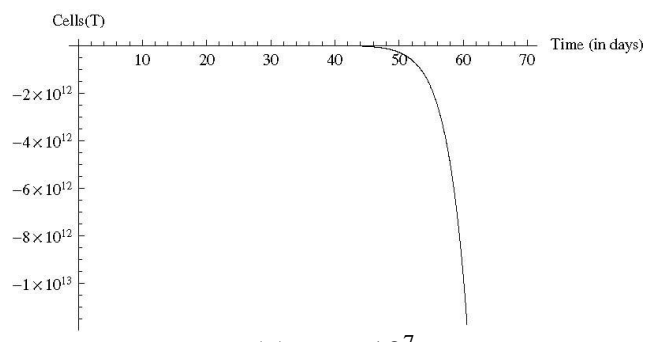

(a) $T_{0}=10^{7}$ 


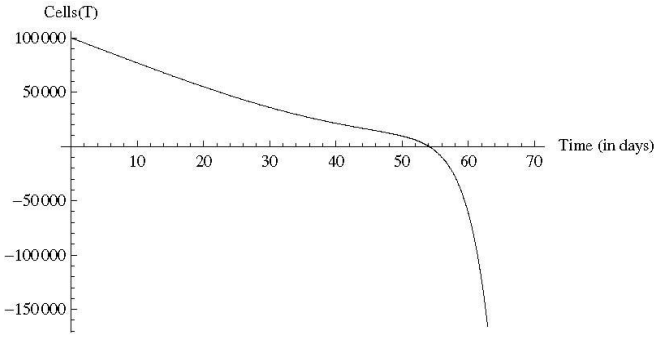

(b) $T_{0}=10^{5}$

Figure 4: Plot of (a) large population, (b) small population of tumor cell vs. time $t$ for DTM, when $v_{0}=0.007$.

Thus, in accordance with this calculation we see that both large tumor size and small tumor size can be removed rapidly by using small molecule drug such that population of both the killer and helper T-cells must be remain unaffected.

\section{CONCLUSION}

In this paper, we have focused on how a tumor can be removed from the system with administration of small molecule drug. As compared to [12], the advantage of the model is that any size of tumor gets eliminated with small molecule drugs and it also gives a rough estimation of quality and dosage of therapy that would be best supplement of the patient's own protection process against the tumor cells. The essential mathematical results obtained for the system (1)-(4) under the condition (5) are also numerically verified in Mathematica 8.0.

Our model construction and management is completely stationed on the after results and interactions amongst tumor cells, CD8 T-killer cells and CD4 T-helper cells, together with the effect of dosage of small molecule drug on these compartments. Alike other mathematical models, the model proposed in this manuscript, it should be considered with caution due to the suppositions made and the perplexity in the evaluation of the parameter values of the model.

\section{ACKNOWLEDGMENT}

The second author thanks to National Institute of Technology Silchar, Assam, India for providing the financial support under the CPDA grant.

\section{REFERENCES}

1. de Pillis, L., Gu, W., and Radunskaya, A. Mixed immunotherapy and chemotherapy of tumors: modelling, applications and biological interpretations. Journal of Theoretical Biology, Vol. 238(4), pp. 841-862, 2006.

https://doi.org/10.1016/j.jtbi.2005.06.037

2. de Pillis, L. G., Radunskaya, A. E., and Wiseman, C. L. A validated mathematical model of cell-mediated immune response to tumor growth. Cancer research, Vol. 65(17), pp. 7950-7958, 2005.

https://doi.org/10.1158/0008-5472.CAN-05-0564
3. dePillis, L., Gu, W., Fister, K., Head, T., Maples, K., Murugan, A., Neal, T., and Yoshida, K. Chemotherapy for tumors: an analysis of the dynamics and a study of quadratic and linear optimal controls. Mathematical Biosciences, Vol. 209(1), pp. 292-315, 2007.

https://doi.org/10.1016/j.mbs.2006.05.003

4. Dhar, B., Gupta, P.K., and Yildirim, A. On solving dynamics of a tumor-immune model with targeted chemotherapeutic drug by multi-step differential transform method. Submitted.

5. Ganguly, R., and Puri, I. Mathematical model for chemotherapeutic drug efficacy in arresting tumour growth based on the cancer stem cell hypothesis. Cell Proliferation, Vol. 40, pp. 338-354, 2006. https://doi.org/10.1111/j.1365-2184.2007.00434.x

6. Ghosh, D., Khajanchi, S., Mangiarotti, S., Denis, F., Dana, S. K., and Letellier, C. How tumor growth can be influenced by delayed interactions between cancer cells and the microenvironment? Biosystems, Vol. 158, 17-30, 2017.

https://doi.org/10.1016/j.biosystems.2017.05.001

7. Gupta, P.K. Approximate analytical solutions of fractional benney-lin equation by reduced differential transform method and homotopy pertubation method. Computers \& Mathematics with Applications, Vol. 61(9), pp. 2829-2842, 2011. https://doi.org/10.1016/j.camwa.2011.03.057

8. Gupta, P.K., and Dhar, B. Dynamical behaviour of fractional order tumor-immune model with targeted chemotherapy treatment. International Journal of Engineering and Technology, Vol. 7(2.28), pp. 6-9, 2018.

https://doi.org/10.14419/ijet.v7i2.28.12872

9. Kirschner, D., and Panetta, J. C. Modeling immunotherapy of the tumor-immune interaction. Journal of Mathematical Biology, Vol. 37(3), pp. 235-252, 1998.

10. Kumari, K., Gupta, P.K., and Shanker, G. An exact solution of diffusion equation with boundary conditions by pade-laplace differential transform method. International Journal of Mathematics and its Applications, Vol. 3(4), pp. 1-8, 2015. https://doi.org/10.9734/PSIJ/2016/23357

11. Kuznetsov, V., A. Makalin, I., A. Taylor, M., and Perelson, A. Nonlinear dynamics of immunogenic tumors: Parameter estimation and global bifurcation analysis. Bulletin of mathematical biology, Vol. 56(4), pp. 295-321, 1994.

12. Liu, P., and Liu, X. Dynamics of a tumor-immune model considering targeted chemotherapy. Chaos, Solitons \& Fractals, Vol. 98, pp. 7-13, 2017.

13. Odibat, Z., Bertelle, C., Aziz-Alaouni, M., and Duchamp, G. A multi-step differential transform method and application to non-chaotic or chaotic systems. Computer and mathematics with applications, Vol. 59(4), pp. 1462-1472, 2010. https://doi.org/10.1016/j.camwa.2009.11.005 
14. Sharma, S., and Samanta, G. Dynamical bhaviour of a tumor-immune system with chemotherapy and optimal control. Journal of Nonlinear Dynamics, 2013. https://doi.org/10.1155/2013/608598

15. Sharma, S., and Samanta, G. Analysis of the dynamics of a tumor-immune system with chemotherapy and immunotherapy and quadratic control. Differential Equations and Dynamical Systems, Vol. 24(2), pp. 149-171, 2016.

https://doi.org/10.1007/s12591-015-0250-1

16. Valle, P. A., Starkov, K. E., and Coria, L. N. Global stability and tumor clearance conditions for a cancer chemotherpy system. Communications in Nonlinear Science and Numerical Simulation, Vol. 40, pp. 206-215, 2016.

https://doi.org/10.1016/j.cnsns.2016.04.025 